\title{
Deutsch-dänische Stereotypenwelten im SMiK-Projekt
}

\author{
Annika Hofmann (Schleswig) und Erla Hallsteinsdóttir (Odense)
}

\begin{abstract}
In this paper, we will first present the current state of research regarding stereotypes and ideas of Germans and Danes about each other. This paper will then display research approaches of the SMiK-project. In the second half of the paper, the results of the questionnaire survey "Typically German - typically Danish" which reveal current German-Danish stereotypes will be presented. Last but not least, this paper includes a study of stereotypes that are used on German-Danish internet platforms and, thus, offer insights into chosen aspects of the linguistic analyses of the SMiK-project.
\end{abstract}

\section{$1 \quad$ Einleitung}

Das Projekt Nationale Stereotype und Marketingstrategien in der interkulturellen deutschdänischen Kommunikation ${ }^{1}$, abgekürzt SMiK, ist ein durch das INTERREG4A-Programm der EU, die Süddänische Universität Odense und die Christian-Albrechts-Universität zu Kiel finanziertes Forschungsprojekt, das sich die Aufdeckung der aktuellen deutsch-dänischen Stereotype und ihre Nutzbarmachung in der interkulturellen Kommunikation und im Fremdsprachenunterricht zum Ziel gesetzt hat.

In diesem Beitrag werden wir zuerst einen kurzen Überblick über die bisherige Forschung zu deutsch-dänischen Stereotypen und Vorstellungen von Deutschen und Dänen übereinander geben und dann die Forschungsansätze aus dem SMiK-Projekt vorstellen. In der zweiten Beitragshälfte stellen wir die Ergebnisse aus der Fragebogenerhebung „,Typisch deutsch - typisch dänisch“ zu aktuellen deutsch-dänischen Stereotypen dar. Die zweite Beitragshälfte enthält ebenfalls anhand der Analyse von Stereotypen in deutsch-dänischen Internetforen einen Einblick in ausgewählte Aspekte der sprachlichen Analysen im SMiK-Projekt.

\footnotetext{
${ }^{1}$ Das Projekt Nationale Stereotype und Marketingstrategien in der interkulturellen deutsch-dänischen Kommunikation (SMiK; www.stereotypenprojekt.eu [13.02.2016]) wurde gefördert durch INTERREG4A SyddanmarkSchleswig-K.E.R.N. mit Mitteln des Europäischen Fonds für regionale Entwicklung; www.interreg4a.de/wm390752 [13.02.2016].
}

Linguistik online 79, 5/2016 - http://dx.doi.org/10.13092/lo.79.3348

CC by 3.0 


\section{Das Deutschlandbild in Dänemark und das Dänemarkbild in Deutschland}

\subsection{Das Deutschlandbild der Dänen}

Zum Deutschlandbild der Dänen kann auf einen umfassenden Fundus an Untersuchungen zurückgegriffen werden. Bereits vor 20 Jahren war Henningsen der Meinung, dass es zu dänischen Deutschlandbildern so viele Studien gebe, dass er die Forschungslage sogar mit der Vermutung, „daß bereits alles zum Thema gesagt ist“ (Henningsen 1996: 144) kommentiert. Dabei bezieht er sich v. a. auf historisch-analytische Arbeiten zu deutsch-dänischen Beziehungen, deren Ergebnisse zum Deutschlandbild in Dänemark mit dem Satz den grimme tys$k e r^{2}$ zusammengefasst werden können. So bezeichnet Lammers in seiner historischen Übersicht die Beziehung zwischen Deutschland und Dänemark vorsichtig als „eine noch etwas delikate und komplizierte Angelegenheit" (Lammers 2000: 57). Auch Henningsen (1996) spricht von einem historisch bedingten Deutschenhass und illustriert anhand zahlreicher Beispiele das schlechte Ansehen der Deutschen in Dänemark. Andere Untersuchungen beschreiben eine „dänische Furcht vor den Deutschen“ (Henningsen 1991: 17). Durch diese Furcht manifestiere sich ein deutsches Feindbild, das sowohl historisch (cf. Rerup 1996; Ruby 1996; Østergård 1991, 1996) als auch in der „,verbreiteten Unwissenheit über den großen Nachbarn“ (Henningsen 1991) verankert sei. Die Überprüfung dessen, inwieweit das historische Deutschenbild des hässlichen/abscheulichen Deutschen in Dänemark immer noch existiert oder ob es in den letzten Jahren tatsächlich positive Veränderungen im Deutschlandbild der Dänen gegeben hat (cf. Schramm 2010), gehört zu den Zielen des SMiK-Projekts.

Neben diesen historisch orientierten Forschungsarbeiten gibt es einzelne Fragebogenuntersuchungen (cf. Bolten 2006), die mittels Assoziationsfragen Stereotype erheben. Grundsätzlich bestätigen sie das tradierte Deutschlandbild. Darüber hinaus gibt es Arbeiten, die sich der empirischen Textanalyse bedienen. Langer (2000a, 2000b, 2003) stellt in einer Studie zum Deutschlandbild in dänischen Medien fest, ,,daß es in dänischen Mediendiskursen nicht DAS Deutschlandimage gibt“" (Langer 2003: 335), sondern mehrere themen-/kontextspezifische Images, die auf der Basis ,eines vorverhandelten Kontextes und einer kollektiven Erinnerung“ (Langer 2003: 331) existieren. Zu einem ähnlichen Ergebnis kommt Müller in ihrer qualitativen Analyse von Stereotypen in 5.220 dänischen und 161 deutschen Zeitungsartikeln mit Berichterstattung zu EU-Thematik (cf. Müller 2005: 163). Sie erfasst das dänische Selbstbild als ,gesellschaftlich etablierte Vorstellungen“ (Müller 2005: 163) und vergleicht es mit dem deutschen Fremdbild von Dänemark, wobei sie deutliche inhaltliche Unterschiede in den in den Zeitungen thematisierten Bereichen feststellt. Mittels Textanalyse wurde ebenfalls das in der dänischen Literatur (cf. Øhrgaard 1996) und das in Deutschlehrwerken für Schulen vermittelte Deutschen- und Deutschlandbild (,langweilig und grau“ Fink 2003: 486, cf. dazu auch Thomsen/Thomsen 2008) erfasst. Bisherige Untersuchungen bestätigen, dass der Fremdsprachenunterricht einen starken Einfluss auf die Ausbildung von Images über andere Länder ausübt (cf. Thomsen/Thomsen 2008; Schulze 2010).

Insbesondere Analysen von Lehrwerken und Unterrichtsmaterial, Lehrer- und Schülerinterviews (cf. Thomsen/Thomsen 2008), die die Entstehung des Deutschlandbildes in der Schule

\footnotetext{
2 Das dänische Adjektiv grim hat zwei Bedeutungen, die mit ,hässlich` und ,abscheulich` wiedergegeben werden können: Der hässliche/abscheuliche Deutsche.
} 
untersuchen, sowie Arbeiten zur generellen - allgemein als schwierig eingeschätzten - Situation des Unterrichtsfaches Deutsch ${ }^{3}$ (cf. Skovgaard Andersen 2010) bilden daher eine wichtige Grundlage für die Einordnung und Interpretation der Ergebnisse zu aktuellen deutschdänischen Stereotypen.

\subsection{Das Dänemarkbild der Deutschen}

Die ,gegenseitige Wahrnehmung von Deutschen und Dänen ist die einer extremen Asymmetrie“" (Henningsen 1996: 141), die sich laut Henningsen einerseits in einem negativ geprägten Deutschlandbild in Dänemark (cf. oben) und andererseits in Deutschland in „Unwissenheit und Desinteresse am nördlichen Nachbarn“ (Henningsen 1996: 142) zeige. Die Deutschen kennen, so Henningsen, nur einige wenige „Kulturdaten“ (Henningsen 1996: 142) über Dänemark. Diese seien insbesondere der Schriftsteller Hans Christian Andersen, die Spielzeugmarke LEGO, die Olsenbande, die kleine Meerjungfrau und die dänische Gemütlichkeit ${ }^{4}$. Darüber hinaus nimmt Henningsen an, dass die Deutschen wenig über die Dänen wissen 5 : „Nicht Antipathie, nicht Sympathie - Desinteresse kennzeichnet den öffentlichen Diskurs.“ (Henningsen 1996: 142).

Im Jahr 2007 wurde ein von der dänischen Regierung in Auftrag gegebener Aktionsplan für die globale Vermarktung von Dänemark veröffentlicht (cf. ReD Associates 2006). Das zusammenfassende Ergebnis zur Bekanntheit von Dänemark in der Welt, das u. a. auf qualitativen Interviews, groß angelegten Umfragen6, Medienanalysen und Expertenmeinungen basiert, stimmt mit Henningsens Ausführungen überein:

Research shows that Denmark is not very well known in most parts of the world and that its image abroad is unclear. However, when people do have an idea of Denmark, their general attitude is positive.

(Ministry for Economic and Business Affairs 2007: 12)

Die dänische Ausgabe der Studie (cf. ReD Associates 2006: 62) enthält eine kurze Darstellung des ermittelten deutschen Dänemarkbilds, das eher widersprüchlich und vage ausfällt. In Deutschland werde Dänemark demnach gleichzeitig als Hersteller von Nischenprodukten von hoher technologischer Qualität und als ein Land mit viel landwirtschaftlicher Lebensmittelproduktion wahrgenommen. Das Land habe einerseits eine stabile und flexible Demokratie, verschließe sich andererseits jedoch immer mehr von der restlichen Welt und sei zunehmend ausländerfeindlicher. Die dänische Gesellschaft werde zugleich sowohl als eine eher konser-

\footnotetext{
3 Entsprechende Untersuchungen zum Dänischen als Schulfach in (Nord-)Deutschland gibt es kaum.

${ }^{4}$ Das deutsche Wort Gemütlichkeit ist nicht äquivalent zum dänischen hygge, es wird jedoch in der Literatur und von vielen (deutschen und dänischen) Sprechern (cf. die Fragebogenantworten in Hallsteinsdóttir 2015a) als Äquivalent verwendet.

${ }^{5}$ Diese Annahme wurde in einer im Projekt ausgearbeiteten Magisterarbeit bestätigt (cf. Meyer 2014: 40), in der in den beiden Universitätsstädten Stuttgart und Flensburg deutsche Studierende nach ihren Vorstellungen über Dänemark befragt wurden. Insbesondere in Stuttgart war es schwierig, Teilnehmende zu gewinnen, denn viele Studierende lehnten die Teilnahme mit der Begründung ab, sie würden nicht genug bzw. gar nichts über Dänemark wissen, und daher könnten sie die Fragen nicht sinnvoll beantworten.

${ }^{6}$ Diese basieren auf Daten vom Anholt Nation Brands Index im 2. Quartal 2006, cf. www.simonanholt.com/Research/research-introduction.aspx [04.10.2015].
} 
vative als auch als eine experimentierfreudige Gesellschaft aufgefasst, die neue Möglichkeiten erforsche und ihre Strukturen dynamisch anpasse.

\section{$3 \quad$ Aktuelle deutsch-dänische Stereotypenwelten}

Als Arbeitsdefinition von Stereotypen wurde im SMiK-Projekt eine sehr offene Definition gewählt: Stereotype werden hier als durch Sprache und Kultur vorgegebene Muster für unser Denken und Handeln aufgefasst, die uns helfen, uns in einer komplexen Welt zurechtzufinden. Stereotype bestimmen unsere positiven und negativen Erwartungen, Vorurteile und Vorstellungen von uns selbst und von anderen. Sie bieten eine erste Orientierung dafür, wie wir die Welt kategorisieren und wie wir uns selbst und andere in unseren Weltansichten einordnen und bilden somit eine wichtige Grundlage für die Begegnung und Interaktion mit anderen Kulturen. Es können drei Arten von Stereotypen unterschieden werden (cf. Bolten 2007):

- Autostereotype: die stereotypen Vorstellungen, die wir über uns selbst haben.

- Heterostereotype: die stereotypen Vorstellungen, die wir über andere haben.

- Meta-/Spiegelstereotype: die stereotypen Vorstellungen, von denen wir glauben, dass andere sie über uns haben.

Gegenstand der Untersuchungen im SMiK-Projekt sind insbesondere die nationalen deutschdänischen Heterostereotype. Die anderen Arten werden zudem teilweise in die Ratgeber- und Unterrichtsmaterialien einbezogen.

Für die Beschreibung der aktuellen deutsch-dänischen nationalen Stereotype wurden im SMiK-Projekt unterschiedliche Methoden eingesetzt, um möglichst viele Aspekte und Konstituierungsformen der Stereotype zu erfassen. Die folgende Auflistung gibt eine kurze Übersicht über die im Projekt durchgeführten Untersuchungen (einen guten Überblick über die Untersuchungen des SMiK-Projekts geben die Poster von der Abschlusskonferenz des Projekts, cf. Hallsteinsdóttir/Kilian 2015):

1) Interviews: In einer der wissenschaftlichen Arbeit vorangestellten Bedarfsanalyse wurden Leitfadeninterviews eingesetzt. Die so gewonnenen Interviewdaten wurden einer detaillierten wissenschaftlichen Analyse unterzogen und auf das Vorkommen von stereotypen Kategorisierungen und Erklärungsmodellen untersucht. (cf. Müller/Hallsteinsdóttir 2016, in diesem Heft; Müller 2016).

2) Korpusanalysen: Für eine Analyse der Versprachlichung von deutschen und dänischen Heterostereotypen wurden ein deutsches und ein dänisches Stereotypenkorpus erstellt. Die Korpora beinhalten alle Wörter und die dazugehörenden Beispielsätze aus dem dänischen und dem deutschen Korpus des Leipziger Wortschatzprojekts, in denen das Morphem \{tysk\} bzw. \{dän\} vorkommt (cf. Hallsteinsdóttir 2015b; Quasthoff/Hallsteinsdóttir in diesem Heft).

3) Textanalysen: Zu kritischen Ereignissen in ausgewählten deutschen und dänischen Zeitungen und Internetforen wurden Analysen in eigens für das SMiK-Projekt aufbereiteten Korpora am Dresdner Center for Digital Linguistics durchgeführt (cf. Hallsteinsdóttir/Kilian 2015). 
4) Fragebogenuntersuchungen zu Kulturdimensionen: Mit geschlossenen Fragen wurden ausgewählte Aspekte von Kulturdimensionen innerhalb von abgegrenzten soziokulturellen Gruppen bzw. Berufsbereichen erfasst. Die Datenauswertung umfasst die Beschreibung von deutsch-dänischen Ähnlichkeiten und Unterschieden (cf. Vandermeeren 2013, 2016; Vandermeeren/Hofmann 2015, 2016). Zusätzlich zu den geschlossenen Fragen wurden offene Fragen zum Einfluss von Hetero- und Meta-/Spiegelstereotypen in deutsch-dänischen Wirtschaftsbeziehungen eingesetzt.

5) Fragebogenuntersuchungen zu ,Typisch deutsch - typisch dänisch“: Um aktuelle und ggf. neue Stereotype und mögliche Veränderungen im Stereotypenbild aufzudecken, wurden Fragebogen mit offenen Fragen verwendet. In diesen wurden Fragen zu unterschiedlichen Aspekten von Land und Leuten gestellt, um möglichst viele Aspekte der deutsch-dänischen Heterostereotype zu erfassen (cf. Hallsteinsdóttir 2015a; Müller/Hallsteinsdóttir 2016).

Für die Erfassung, Analyse und Beschreibung von Stereotypen mit diesem Methodenpluralismus werden drei Ebenen der Manifestation von Sprache und Kultur unterschieden, in die sich die Daten des Projekts einordnen lassen (cf. ausführlich zu dieser Dreiteilung in z. B. Hallsteinsdóttir 2013; Hallsteinsdóttir/Farø 2010; Kilian 2015):

- Ebene des Sprachsystems: Stereotype im Wortschatz können u. a. durch die Analyse von Lehrwerken, Wörterbuchdaten (cf. Heier 2016) und in Publikationen zu bisherigen Untersuchungen erfasst werden. Hier geht es um die Frage, welche Stereotype konventionell in Verbindung mit lexikalischen Einheiten in der deutschen/dänischen Sprache und Kultur verankert sind. Hier werden Fragen herangezogen wie: Welche Stereotype stehen über Lexikalisierungen im Wortschatz in Wörterbüchern für den Sprachgebrauch (Realisierung in Texten) zur Verfügung? Welche Stereotype vermitteln die Lehrwerke für Deutsch/Dänisch als Fremdsprache für dänische und deutsche Lerner?

- Ebene des Sprachgebrauchs: Die Realisierung von Stereotypen in der sprachlichen Kommunikation kann u. a. durch die Analyse von Interviewdaten und Sprachdaten in Korpora auf der Wortschatz- und Textebene erfasst werden. Im SMiK-Projekt handelt es sich einerseits um Korpusdaten des Leipziger Wortschatzprojekts und andererseits um Korpora mit Texten aus ausgewählten Medien, die am Dresden Center for Digital Linguistics spezifisch für das Projekt zusammengestellt wurden. Die Interviewdaten des SMiKProjekts (cf. Müller 2016) erfüllen zwar kommunikative Funktionen eines Interviews. Inhaltlich beziehen sich die Interviews aber auf eine Metaebene zu deutsch-dänischen Aspekten. Die Interviewdaten geben daher Aufschluss sowohl über die Kompetenz der Sprecher als auch über Stereotype im Sprachgebrauch. ${ }^{7}$

- Ebene der Sprach- und Kulturkompetenz: Stereotype Vorstellungen in den Köpfen der Sprecher können insbesondere durch die Analyse von Fragebogendaten beschrieben werden. Die Fragebogendaten des SMiK-Projekts (cf. Hallsteinsdóttir 2015a; Hofmann/Hallsteinsdóttir in diesem Heft, Müller/Hallsteinsdóttir 2016 sowie Vandermeeren

\footnotetext{
${ }^{7}$ An der Schnittstelle der Sprach- und Kulturkompetenz und dem Sprachgebrauch stellt sich die Frage, ob manche assoziativ-semantischen Stereotype schon so fest mit der sprachlichen Form verknüpft sind, dass die sprachliche Struktur isoliert kaum gebraucht werden kann, ohne das Stereotyp aufzurufen (cf. Kilian 2001, 2003 sowie eine persönliche Mitteilung von Jörg Kilian).
} 
2016) können als Versprachlichungen mentaler Konzepte betrachtet werden. Sie sind kein kommunikativer Sprachgebrauch im herkömmlichen Sinne, sondern mehr oder weniger reflektierte metasprachliche Akte. Als solche können sie als Indizien für die Beschaffenheit einer sprachlich-kulturellen Kompetenz gedeutet werden. Die Resultate der Fragebogenerhebungen geben Antworten auf Fragen wie: Welche Stereotype existieren in der Sprach- und Kulturkompetenz der Teilnehmer? Welche vorgefertigten Bilder haben sie von den Deutschen bzw. den Däninnen und Dänen? Welche Wörter und assoziative Vorstellungen benutzen sie über Deutsche bzw. Däninnen und Dänen?

Realisiert im Sinne von als in der wirklichen Welt unabhängig vom SMiK-Projekt existierendes Phänomen sind im Grunde genommen nur die authentischen Produkte des Sprachgebrauchs, die mithilfe von beständigen Medien kodiert sind (als Texte im weiten Sinne) und unverändert beliebig häufig rezipiert werden können. Die beiden anderen hier angenommenen Konstituierungsformen von Sprache können linguistisch zwar auch beschrieben werden, ihre Unbeständigkeit als abstraktes bzw. kognitives Phänomen macht aber ihre Erfassung und intersubjektive Überprüfung kompliziert.

Im Folgenden werden die Ergebnisse aus der Fragebogenuntersuchung zu „typisch deutsch typisch dänisch“ und aus den Textanalysen zu kritischen Ereignissen dargestellt und diskutiert.

\section{Die Fragebogenuntersuchung „Typisch deutsch - typisch dänisch?“}

Die Fragebogenuntersuchung „Typisch deutsch - typisch dänisch“ wurde von August 2013 bis März 2014 online durchgeführt. Insgesamt wurden 506 dänische und 558 deutsche Fragebogen vollständig ausgefüllt und ausgewertet (cf. ausführlich in Hallsteinsdóttir 2015a; Müller/Hallsteinsdóttir 2016). Neben den offenen Fragen zum Typischen wurden Metadaten zum Geschlecht, Alter, Wohnort, zur Ausbildung, zu Sprachkenntnissen und Kenntnissen über Deutschland (dänische Teilnehmende) bzw. Dänemark (deutsche Teilnehmende) erfasst.

\subsection{Metadaten zu den Teilnehmenden}

Knapp zwei Drittel (298 bzw. 59 \%) der dänischen Fragebogen wurden von Frauen und gut ein Drittel (189 bzw. 37 \%) von Männern ausgefüllt. Das Durchschnittsalter der Befragten beträgt 30,9 Jahre. Von den 506 Teilnehmenden wohnen 467 (92\%) in Dänemark und 16 (3\%) in Deutschland. 456 (90\%) Zielpersonen haben die dänische Nationalität und 245 (48 \%) geben an, nur einen Schulabschluss zu haben. Über die Hälfte (267 bzw. 53 \%) der Teilnehmenden hat einen Hochschulabschluss, 68 (13\%) haben eine Berufsausbildung und $66(13 \%)$ eine andere Ausbildung.

Die Teilnehmenden in der deutschsprachigen Fragebogenerhebung weisen eine ähnliche Geschlechterverteilung auf: 320 (57 \%) der Befragten sind Frauen und 215 (39\%) sind Männer. Das Durchschnittsalter ist jedoch 10 Jahre höher, die deutschen Teilnehmenden sind im Durchschnitt 40,11 Jahre alt. 510 (91\%) befragte Personen haben die deutsche Nationalität, jedoch wohnen nur 459 (82\%) in Deutschland (dafür aber 56 bzw. $10 \%$ in Dänemark). Als Ausbildung geben 311 (56\%) einen Schulabschluss, 323 (58 \%) einen Hochschulabschluss, $216(39 \%)$ eine Berufsausbildung und 46 (8\%) eine andere Ausbildung an. Der Ausbil- 
dungsstand weicht insbesondere in der Anzahl von Personen mit Berufsausbildung von den Daten der dänischen Teilnehmenden ab.

In den 506 dänischen Fragebogen teilten 468 (93\%) Befragte eine eindeutige Wohnortangabe via einer Postleitzahl in Dänemark mit. Insgesamt leben 192 (37 \%) Teilnehmende in Südjütland. Werden die 143 Antworten von der Insel Fünen dazu gezählt (28 \%), wohnen zwei Drittel $(66 \%)$ der dänischen Teilnehmenden im grenznahen INTERREG4A-Gebiet. 86 (17\%) Befragte geben einen Wohnort auf der Insel Seeland an. Auffällig ist die geringe Anzahl an Antworten aus anderen Gebieten von Jütland (47 Personen bzw. 9 \%).

Von 558 deutschen Fragebogen weisen 448 (80\%) eine eindeutige Wohnortangabe (mit Postleitzahl) in Deutschland auf. Demnach leben 206 (37\%) Teilnehmende in SchleswigHolstein. Werden Hamburg (22) und Mecklenburg-Vorpommern (5) dazu genommen, wohnen insgesamt $42 \%$ der deutschen Teilnehmenden in der Nähe der deutsch-dänischen Grenze. Auffällig ist mit 9\% (50 Personen) die geringe Anzahl an Antworten aus den östlichen Bundesländern inkl. Berlin. 173 Antworten (31\%) verteilen sich dagegen auf die südlichen und westlichen Teile von Deutschland.

\subsection{Kenntnisse der Teilnehmenden über die jeweils andere Nation}

Um zu erfassen, wodurch die Teilnehmenden ihre Kenntnisse über die jeweils andere Nation erworben haben, wurden geschlossene Fragen gestellt, in denen die Teilnehmenden danach gefragt wurden, aus welchen Kontexten sie die Deutschen bzw. die Dänen kennen. Vorgegeben waren die Kontexte privater Kontakt, beruflicher Kontakt, Urlaub, Medien, Schule und Anderes, zu denen die Antwortmöglichkeiten sehr gut, gut, wenig, gar nicht und keine Angabe vorgegeben waren. Die Verteilung der Antworten zu den einzelnen Antwortmöglichkeiten wird in den Abbildungen 1-5 gezeigt.

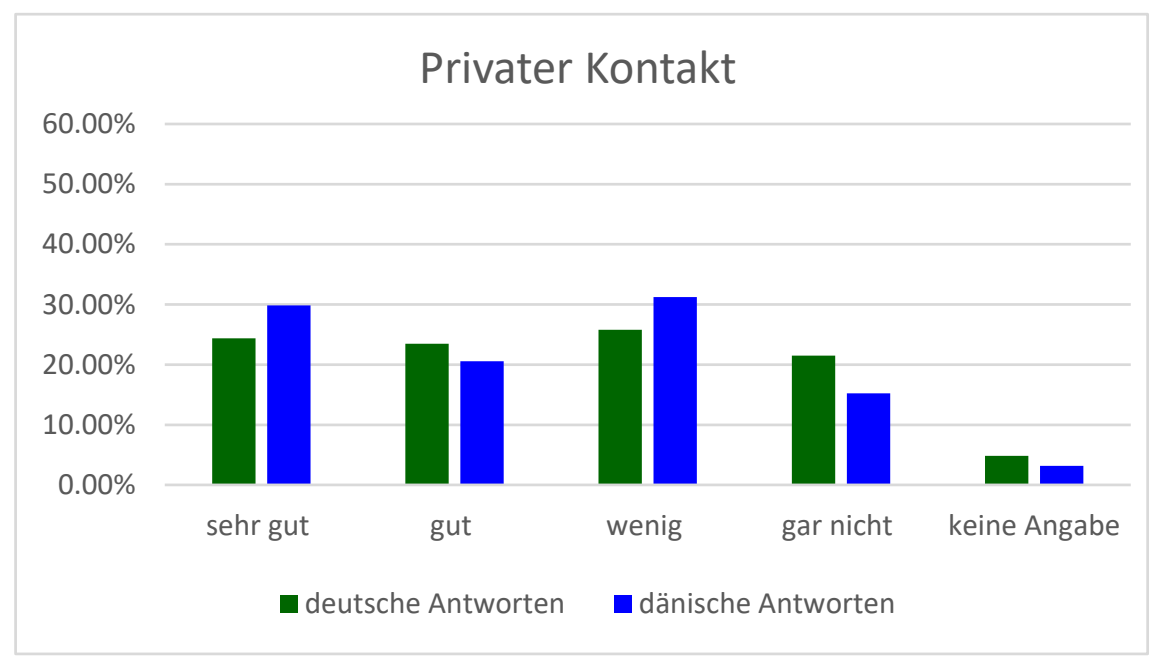

Abbildung 1: Verteilung der Antworten auf die Frage, wie gut die Teilnehmenden die jeweils andere Nation über private Kontakte kennen

Private Kontakte (Abbildung 1): Die Hälfte der deutschen und die Hälfte der dänischen Teilnehmenden kennen die jeweils andere Nationalität sehr gut oder gut (Deutsche: sehr gut (24\%); gut (23\%), Dänen: sehr gut (30\%) oder gut (21\%)) durch private Kontakte. $26 \%$ der deutschen Befragten kennt die Dänen wenig und gut ein Fünftel (22 \%) gar nicht durch 
private Kontakte. Hier gibt es einen geringfügigen Unterschied zu den dänischen Teilnehmenden. Diese kennen die Deutschen mit $31 \%$ wenig und mit $15 \%$ gar nicht durch private Kontakte.

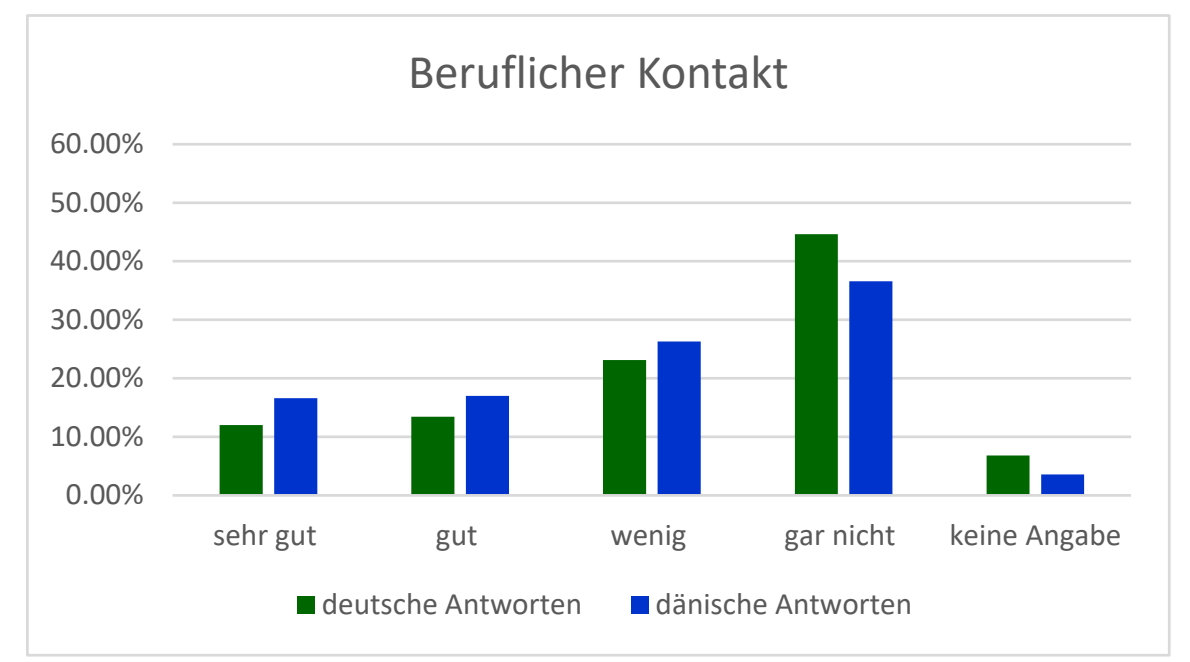

Abbildung 2: Verteilung der Antworten auf die Frage, wie gut die Teilnehmenden die jeweils andere Nation über geschäftliche Kontakte kennen

Berufliche Kontakte (Abbildung 2): Deutlich weniger Teilnehmende kennen die jeweils andere Nation durch berufliche Kontakte als durch private Kontakte. Nur ein Viertel der deutschen Teilnehmenden gibt an, die Dänen sehr gut (12\%) oder gut (13\%) zu kennen. $23 \%$ teilten mit, sie wenig durch beruflichen Kontakt zu kennen. Fast die Hälfte kennt die Dänen gar nicht $(45 \%)$ durch berufliche Kontakte. Ein Drittel der dänischen Teilnehmenden gibt an, die Deutschen sehr gut (17\%) oder gut (17\%) durch Kontakte im Beruf zu kennen. Wenig kennen die Dänen $26 \%$ der deutschen Befragten durch berufliche Kontakte. Gut ein Drittel kennt die Deutschen gar nicht (37\%) durch berufliche Kontakte.

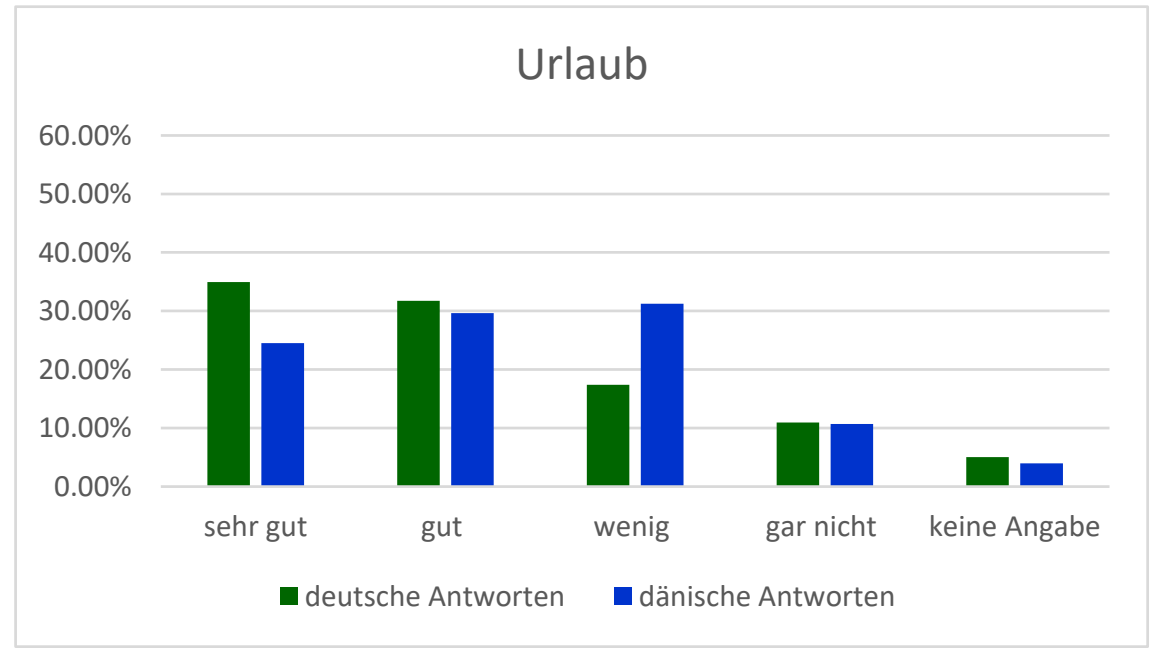

Abbildung 3: Verteilung der Antworten auf die Frage, wie gut die Teilnehmenden die jeweils andere Nation aus dem Urlaub kennen

Urlaub in Dänemark/Deutschland (Abbildung 3): Die überwiegende Anzahl der Teilnehmenden kennt das andere Land aus dem Urlaub. So gaben $35 \%$, $32 \%$ und $17 \%$ der deutschen Teilnehmenden an, Dänemark sehr gut, gut oder wenig aus dem Urlaub in Dänemark zu kennen. Von den dänischen Befragten teilten $25 \%$, $30 \%$ und $31 \%$ mit, Deutschland aus 
dem Urlaub sehr gut, gut oder wenig zu kennen. Insgesamt sind es $84 \%$ der deutschen und $86 \%$ der dänischen Teilnehmenden, die demnach schon einmal Urlaub im jeweils anderen Land gemacht haben. Nur $11 \%$ der deutschen und der dänischen Befragten kennen das andere Land gar nicht aus dem Urlaub:

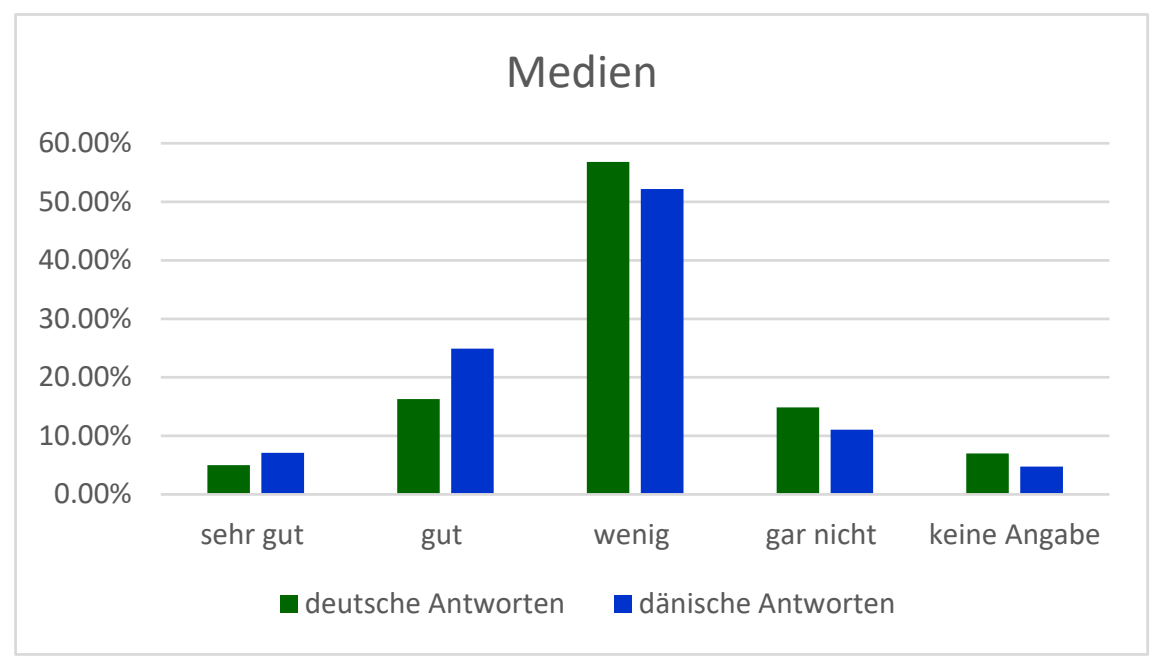

Abbildung 4: Verteilung der Antworten auf die Frage, wie gut die Teilnehmenden die jeweils andere Nation aus den Medien kennen

Medien (Abbildung 4): Nach der Selbsteinschätzung der Befragten haben die Medien nur einen geringen Einfluss auf die Kenntnisse über Deutschland bzw. Dänemark. So spielen die Medien für die deutschen Teilnehmenden keine allzu große Rolle für die Kenntnis des dänischen Nachbarn, denn nur $5 \%$ kennt Dänemark sehr gut und $16 \%$ gut aus den deutschen Medien. $57 \%$ bzw. $15 \%$ der Zielpersonen geben an, Dänemark wenig oder gar nicht aus den Medien zu kennen. Auf der anderen Seite kennen die Dänen Deutschland geringfügig besser aus den Medien. Aber auch hier sind es nur $7 \%$ bzw. $25 \%$, die Deutschland nach eigenen Angaben sehr gut bzw. gut aus den dänischen Medien kennen. $52 \%$ der Befragten gibt an, Deutschland wenig aus den Medien zu kennen. $11 \%$ kennt das Land nach eigener Mitteilung gar nicht durch die Medien.

Schule (Abbildung 5): Bei den Angaben dazu, wie gut die Befragten das andere Land aus der Schule kennen, treten durch die Vorgaben des Ausbildungssystems zu erwartende Unterschiede auf. Nur $11 \%$ bzw. $6 \%$ der deutschen Befragten kennt Dänemark sehr gut bzw. gut durch die Schule, was ggf. z. T. darauf zurückgeführt werden kann, dass Dänisch nur an wenigen deutschen Schulen unterrichtet wird. Ein Viertel der Befragten (25\%) gibt wenig an und gut die Hälfte (51\%) gar nicht. Deutsch ist dagegen ein Unterrichtsfach (seit zwei Jahren ab der 5. Klasse, vorher ab der 7. Klasse) in den dänischen Volksschulen ${ }^{8}$ und in den gymnasialen Ausbildungen. Dies spiegelt sich darin wider, dass $20 \%$ der Dänen Deutschland sehr gut und $43 \%$ Deutschland gut durch die Schule kennt. Gut ein Viertel (28\%) gibt wenig an und nur $5 \%$ kennt Deutschland gar nicht aus der Schule.

\footnotetext{
${ }^{8}$ Dän. folkeskole: eine Einheitsschule, die alle Schülerinnen und Schüler die ersten neun Schuljahre besuchen.
} 


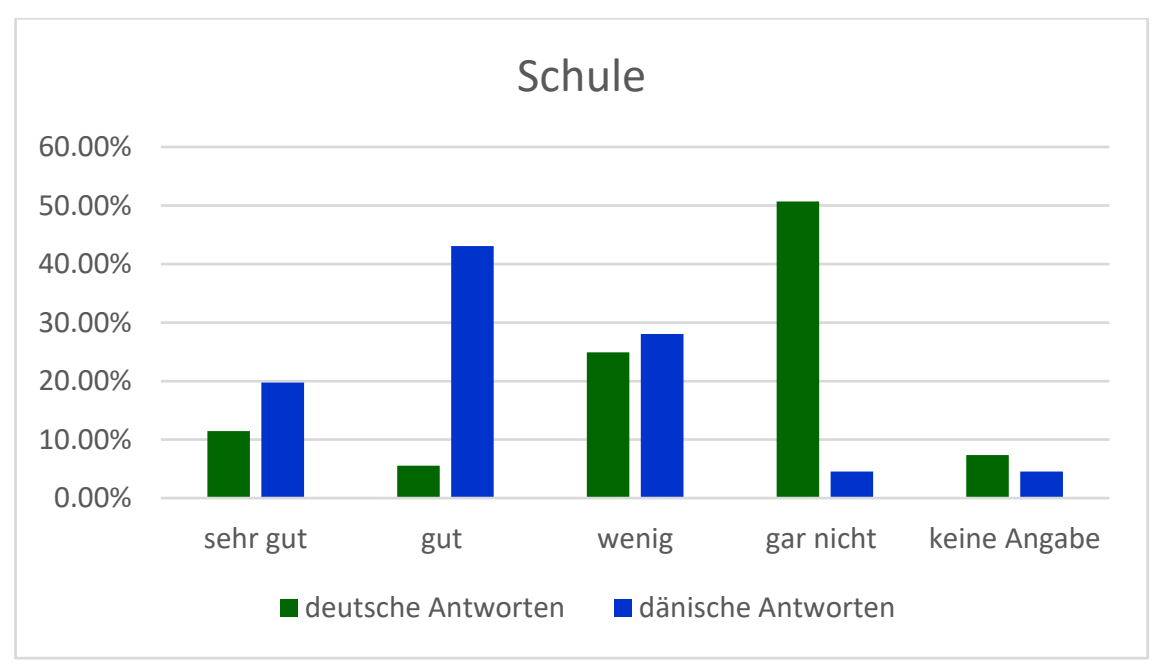

Abbildung 5: Verteilung der Antworten auf die Frage, wie gut die Teilnehmenden die jeweils andere Nation aus der Schule kennen

Die Auswertung der Antworten zu der Frage, aus welchen Kontexten die Teilnehmenden Deutsche bzw. Dänen kennen, zeigt, dass die Kenntnisse insbesondere privater Natur sind, d. h. durch private Kontakte und Urlaub im anderen Land entstanden sind. Bei den dänischen Teilnehmenden spielt die Schule zudem eine große Rolle für die Vermittlung von Wissen über Deutschland. Auffallend ist der als gering angegebene Einfluss der Medien auf das gegenseitige Wissen übereinander. Der angenommene geringe Einfluss der Medien ist insbesondere deshalb interessant, weil viele Untersuchungen zu nationalen Stereotypen sowie Länderbildern/-images auf der Analyse von Medien(texten) basieren, so auch die Textanalysen im SMiK-Projekt, und weil zudem angenommen wird, dass Medien einen großen Einfluss auf die Bildung und Tradierung von Stereotypen haben (cf. z. B. Schulze 2010). Unsere Daten zeigen hier allerdings nur die (bewusste) Selbsteinschätzung der Teilnehmenden, sie können daher kein Maßstab für den nicht wahrgenommenen (unbewussten) Einfluss der Medien auf das gegenseitige Wissen und die stereotypen Vorstellungen der Deutschen und der Dänen übereinander sein.

\subsection{Sprachkenntnisse}

Die teilnehmenden Zielpersonen wurden gebeten, auf einer Skala von 1 bis 10 anzugeben, wie gut sie die deutsche bzw. die dänische Sprache beherrschen $(1=$ gar nicht, $10=$ Muttersprache). Die Abbildungen 6 und 7 zeigen die Deutschkenntnisse der dänischen und die Dänischkenntnisse der deutschen Teilnehmenden: 


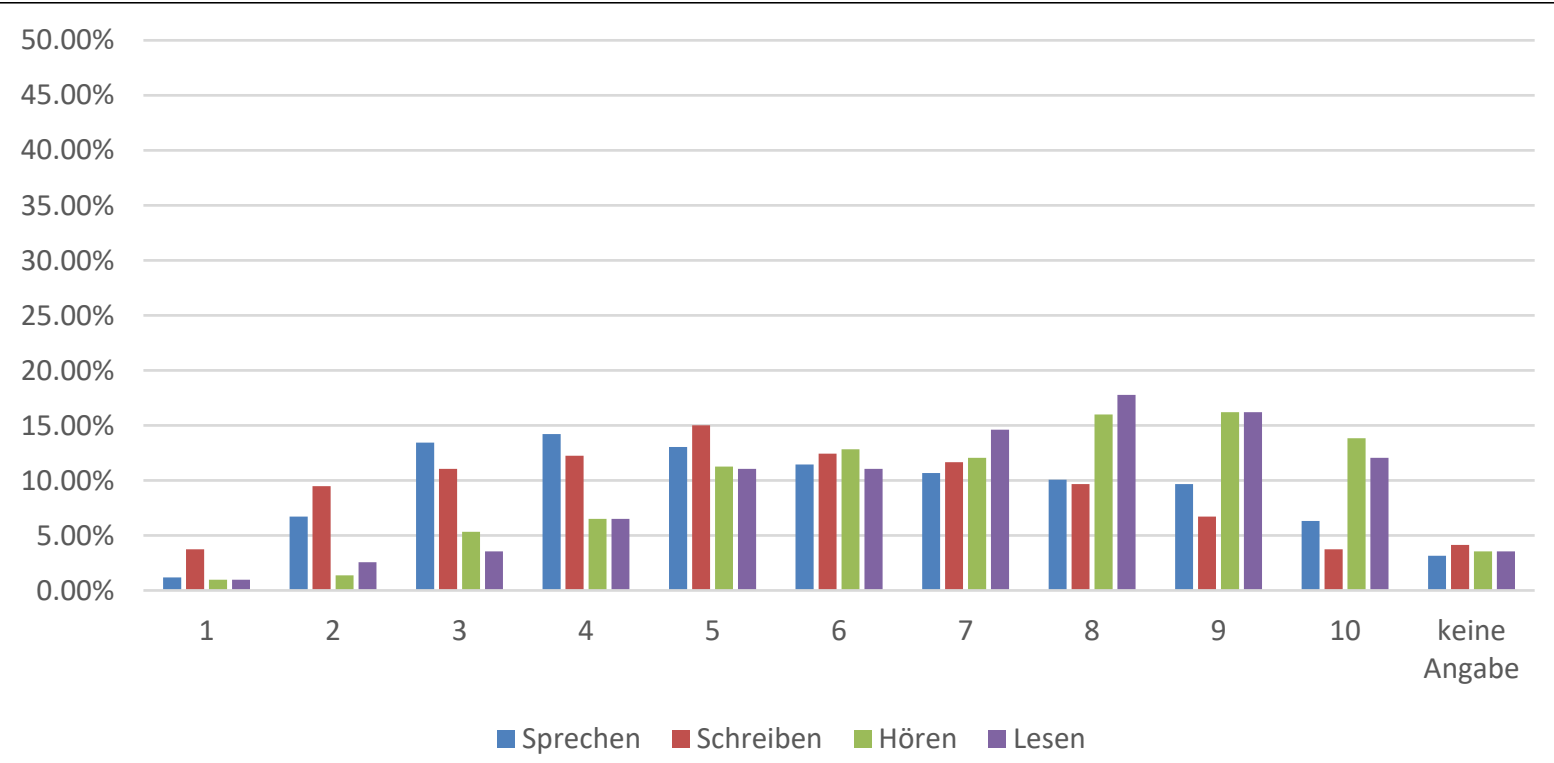

Abbildung 6: Selbsteinschätzung der Deutschkenntnisse der dänischen Teilnehmenden

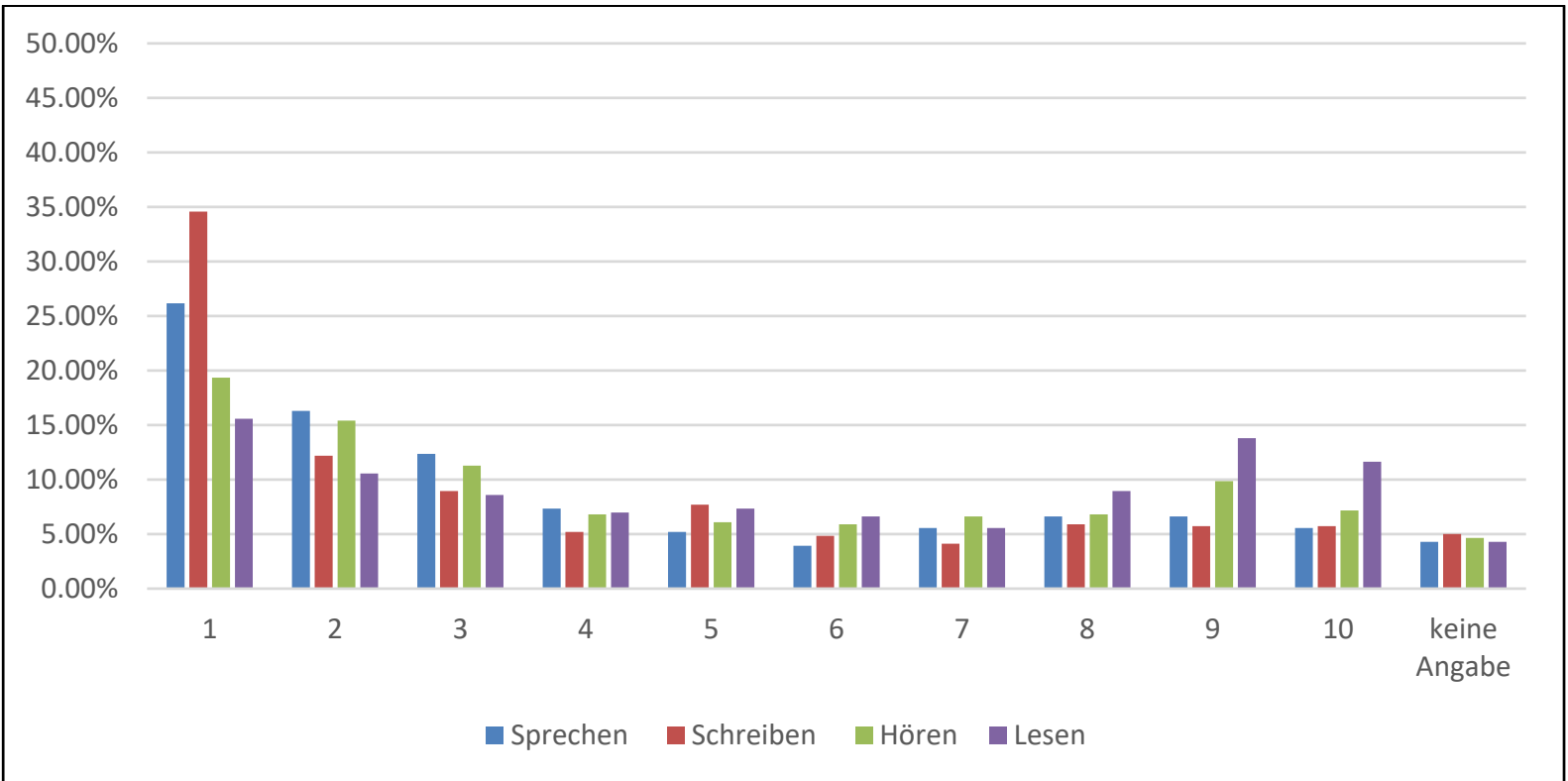

Abbildung 7: Selbsteinschätzung der Dänischkenntnisse der deutschen Teilnehmenden

In Tabelle 1 werden die Sprachkenntnisse auf der Skala 1 bis 10 in die drei Bereiche wenig (2-4), mittel (5-7) und gut (8-10) zusammengefasst, um die Verteilung für die angegebenen Sprachkenntnisse besser vergleichen zu können:

\begin{tabular}{|c|c|c|c|c|c|c|c|c|}
\hline & \multicolumn{2}{|c|}{$\begin{array}{l}\text { mündliche Sprach- } \\
\text { fertigkeit }\end{array}$} & \multicolumn{2}{|c|}{$\begin{array}{l}\text { schriftliche Sprach- } \\
\text { fertigkeit }\end{array}$} & \multicolumn{2}{|c|}{$\begin{array}{l}\text { Sprachverstehen (Hö- } \\
\text { ren) }\end{array}$} & \multicolumn{2}{|c|}{$\begin{array}{l}\text { Sprachverstehen } \\
\text { (Lesen) }\end{array}$} \\
\hline & $\begin{array}{l}\text { Dänisch- } \\
\text { kenntnisse }\end{array}$ & $\begin{array}{l}\text { Deutsch- } \\
\text { kenntnisse }\end{array}$ & $\begin{array}{l}\text { Dänisch- } \\
\text { kenntnisse }\end{array}$ & $\begin{array}{l}\text { Deutsch- } \\
\text { kenntnisse }\end{array}$ & $\begin{array}{l}\text { Dänisch- } \\
\text { kenntnisse }\end{array}$ & $\begin{array}{l}\text { Deutsch- } \\
\text { kenntnisse }\end{array}$ & $\begin{array}{l}\text { Dänisch- } \\
\text { kenntnisse }\end{array}$ & $\begin{array}{l}\text { Deutsch- } \\
\text { kenntnisse }\end{array}$ \\
\hline $\begin{array}{l}\text { Gar } \\
\text { nicht (1) }\end{array}$ & 26,2 & 1,2 & 34,6 & 3,8 & 19,4 & 1,0 & 15,6 & 1,0 \\
\hline $\begin{array}{l}\text { Wenig } \\
(2-4)\end{array}$ & 36,0 & 34,4 & 26,3 & 32,8 & 33,5 & 13,2 & 26,2 & 12,7 \\
\hline
\end{tabular}




\begin{tabular}{|l|c|c|c|c|c|c|c|c|}
\hline $\begin{array}{l}\text { Mittel } \\
(5-7)\end{array}$ & 14,7 & 35,2 & 16,7 & 39,1 & 18,6 & 36,2 & 19,5 & 36,8 \\
\hline $\begin{array}{l}\text { Gut (8- } \\
10)\end{array}$ & 18,8 & 26,1 & 17,4 & 20,2 & 23,8 & 46,0 & 34,4 & 46,0 \\
\hline
\end{tabular}

Tabelle 1: Verteilung der Sprachkenntnisse der deutschen und der dänischen Teilnehmenden

Die Selbsteinschätzung der Dänen zeigt, dass sie - bis auf $1 \%$ - grundsätzlich Deutschkenntnisse haben. Sie schätzen ihr Sprachverstehen (Hören und Lesen) im Deutschen tendenziell als eher gut ein, während sie ihre Schreib- und Sprechfertigkeiten als schlecht oder mittelmäBig beurteilen. Für das Sprachverstehen, d. h. die rezeptiven Sprachfertigkeiten, gibt insgesamt knapp die Hälfte (46 \%) die hohen Werte 8-10 an. Jeweils nur $13 \%$ der befragten Personen teilten die niedrigen Werte 2-4 jeweils für das Hören und Lesen mit. Bei den produktiven Sprachfertigkeiten ist die Verteilung der Sprachkenntnisse umgekehrt, denn hier geben nur jeweils $26 \%$ (Sprechen) und $20 \%$ (Schreiben) die Werte 8-10 an, während $34 \%$ (Sprechen) und $33 \%$ (Schreiben) der Teilnehmenden die Werte 2-4 angeben.

Die Selbsteinschätzung der Deutschen zeigt, dass etwa zwei Drittel ihre Sprechfertigkeit im Dänischen tendenziell als nicht vorhanden oder gering einschätzen. Die deutschen Teilnehmenden schätzen ihr Sprachverstehen (Hören) im Dänischen etwa zur Hälfte als nicht vorhanden oder gering und zur Hälfte als mittelmäßig oder gut ein. Nur $16 \%$ der Personen können gar kein Dänisch lesen. Gut ein Drittel gibt sogar gute und sehr gute Kenntnisse (Werte 8-10) an. Insgesamt teilten 80 \% mit, Lesefertigkeiten für Dänisch zu haben. Demnach haben nach eigenen Angaben nur $16 \%$ der deutschen Teilnehmenden gar keine Dänischkenntnisse.

Insgesamt können wir auf der Basis der Metadaten schlussfolgern, dass die Teilnehmenden in der Fragebogenuntersuchung über recht gute Kenntnisse über das andere Land sowie eher gute Sprachkenntnisse in der jeweiligen Fremdsprache Deutsch bzw. Dänisch verfügen. Wir gehen daher davon aus, dass Henningsens (1996: 142) Beobachtung einer deutschen Unwissenheit über Dänemark sowie eines Desinteresses von Deutschen gegenüber Dänemark und den Dänen nicht für unsere Befragten kennzeichnend ist. Den meisten Antworten liegen vielmehr eigene Erfahrungen mit dem anderen Land und dessen Bewohnern zugrunde.

\subsection{Offene Fragen zu „Typisch deutsch - typisch dänisch?““}

Um herauszufinden, welche Stereotype in den Köpfen der Menschen über Deutschland/die Deutschen und Dänemark/die Däninnen/Dänen vorhanden ist, wurden folgende Fragen gestellt:

1) Schreiben Sie bitte die ersten drei Wörter auf, die Ihnen zu Dänemark/Deutschland einfallen.

2a) Was kennzeichnet eine typische Dänin/Deutsche?

2b) Was kennzeichnet einen typischen Dänen/Deutschen?

3a) Was ist für Sie typisch dänisch/deutsch? - (eher) positiv

3b) Was ist für Sie typisch dänisch/deutsch? - (eher) negativ 
4) Eine große dänische/deutsche Firma bittet Sie, eine Werbekampagne in Deutschland/Dänemark zu machen. Welche typischen dänischen Eigenschaften würden Sie in einer solchen Kampagne einsetzen, um ein dänisches/deutsches Produkt in Deutschland/Dänemark zu verkaufen? Sie können die Frage gerne mit der Angabe eines konkreten Produktes beantworten.

Während Frage 1 assoziatives Wissen u. a. in Form von Faktenwissen, kulturellem und historischem Wissen erfragt, bezieht sich Frage 2 auf die Auffassung über die Menschen aus dem jeweiligen Land. Hier wird erfasst, welche Eigenschaften und Phänomene die Teilnehmenden mit Däninnen und Dänen bzw. Deutschen verbinden. Bei Frage 3a und 3b stehen persönliche Einstellungen zu typischen Eigenschaften im Vordergrund. Mit diesen Fragen werden die subjektiven - positiven und negativen - Wertungen des typisch Deutschen bzw. Dänischen erfasst.

Im Folgenden werden die Ergebnisse der Datenauswertung dargelegt.

\subsubsection{Assoziationen mit Deutschland und Dänemark}

Bei der ersten Frage sollten die Teilnehmenden die ersten drei Wörter aufschreiben, die ihnen zu Deutschland bzw. Dänemark einfallen. Mit einer ähnlichen Frage haben Bolten/Weißflog (cf. Bolten 2006; Bolten/Weißflog 2005) in einer groß angelegten Studie Stereotype über Deutschland in 21 Ländern, darunter auch Dänemark, untersucht.

Die Auswertung der dänischen Daten zeigt, dass Berlin, Würstchen (dän. pølser, in verschiedenen Variationen), Bier, Hitler, der Zweite Weltkrieg, Fußball und Autos am häufigsten genannt werden. Weiterhin gibt es ein häufiges Vorkommen von Wörtern wie (Angela) Merkel, Ordnung, Grenzhandel, Sprache, Autobahn, Süßigkeiten, Kultur und Oktoberfest. Hier korrelieren einige Begriffe (aus den Themenbereichen Zweiter Weltkrieg, Sport und Politik) mit den in den Korpusanalysen des Projekts festgestellten signifikanten Kookkurrenzen zu *tysk*Wörtern in den dänischen Korpora (Medientexte, cf. Quasthoff/Hallsteinsdóttir in diesem Heft). Das häufige Nennen der deutschen Sprache deutet auf einen Zusammenhang zwischen Deutsch als Unterrichtsfach und entsprechenden Kenntnissen über Deutschland aus der Schule (cf. Abbildung 5) und Sprachkenntnissen der Teilnehmenden hin (cf. Abbildung 6). Die Sprache wird in den Antworten jedoch nicht unbedingt als positiv bewertet (cf. ausführlich in Müller 2016).

In den deutschen Daten überwiegen die Begriffe Strand, Kopenhagen, Hotdog und Urlaub sowie Wörter wie Meer, Ruhe, Entspannung, Ferienhaus, freundlich/Freundlichkeit, Natur, Dünen, gemütlich/hyggelig und Dannebrog (die dänische Flagge). In den Nennungen sind einige der dänischen „Kulturdaten“ (Henningsen 1996: 142) zu sehen. Allerdings fehlen hier auch wichtige dieser Kulturdaten wie LEGO und die Olsenbande, andere wie Hans Christian Andersen und die kleine Meerjungfrau werden nur selten genannt. Die häufige Nennung urlaubsbedingter Wörter überrascht nicht, denn wie Abbildung 3 zeigt, kennen $67 \%$ der deutschen Teilnehmenden Dänemark gut oder sehr gut aus dem Urlaub. Auffällig ist aber, dass sich die entsprechenden Urlaubserfahrungen der Dänen nicht so deutlich in den Antworten widerspiegeln. 
In den Ergebnissen der Studie von Bolten (cf. Bolten 2006: 6; Bolten/Weißflog 2005: 52) werden der Zweite Weltkrieg (18), Mauer Berlin (8), die Wiedervereinigung (8), Bier (7) und Hitler (4) als die häufigsten Stereotype der dänischen Teilnehmenden genannt. Auch wenn der Studie nur eine verhältnismäßig geringe Teilnehmerzahl von 42 (cf. Bolten/Weißflog 2005: 12) zu Grunde liegt, kann festgestellt werden, dass die Ergebnisse eine recht hohe Übereinstimmung in der Nennung des Zweiten Weltkriegs zeigen. In den Antworten auf die erste Frage der SMiK-Untersuchung wird Hitler insgesamt 50-mal genannt und Nennungen zum Ersten und Zweiten Weltkrieg bzw. Krieg kommen 40-mal, und zu Nazi, Nazideutschland, Nazismus 12-mal vor. In den Antworten auf die anderen Fragen, in denen die positiven und negativen Vorstellungen der Teilnehmenden vom typisch Deutschen oder ihre Vorstellungen von den Menschen in Deutschland erfragt werden, kommt der Themenbereich zu Krieg, Nationalsozialismus, Hitler jedoch kaum vor. Wir schließen daraus, dass mit der DreiWörter-Frage nach den Assoziationen zu Deutschland bzw. Dänemark v. a. eine Art assoziatives Fakten-Wissen über das jeweils andere Land erfasst wird. Dieses Wissen ist ein Potenzial, das ggf. die Grundlage für die Entstehung oder Re-Aktivierung von Stereotypen bilden kann, aber als Wissen nicht zwingend als Stereotype im Sinne unserer SMiK-Definition als Denk- und Handlungsmuster aufzufassen ist. Die Ergebnisse unserer Befragung zeigen, dass Assoziationsfragen alleine nicht alle Aspekte von nationalen Stereotypen erfassen können, dafür ist eine deutlich differenziertere Fragestellung unter Einbeziehung unterschiedlicher Aspekte des Nationalen notwendig.

\subsubsection{Typische deutsche und dänische Menschen}

In der zweiten Frage, die in zwei randomisierte Unterfragen aufgeteilt wurde, sollten die Teilnehmenden angeben, was (a) eine typische Dänin/Deutsche und (b) einen typischen Dänen/Deutschen kennzeichnet. Diese Frage zielt auf die Erfassung stereotyper Vorstellungen über die Menschen im jeweils anderen Land.

Die Fragen 2 und 3 zeigen, dass die deutschen Befragten vor allem die Eigenschaften freundlich (auch nett; 216 Nennungen) und blond (286 Nennungen) sowohl mit Däninnen als auch mit Dänen verbinden. Weitere häufig genannte Eigenschaften sind hübsch (auch attraktiv, gutaussehend, schön; 64 Nennungen), modisch (auch modebewusst, modern, gut gekleidet, stylisch; 58 Nennungen) und offen (auch aufgeschlossen; 51 Nennungen) über die Däninnen sowie gelassen (auch entspannt; 62 Nennungen), ebenso offen (auch aufgeschlossen, 40 Nennungen) und groß (36 Nennungen) über die Dänen. Auch werden Eigenschaften wie selbstbewusst, blauäugig und schlank über die Däninnen und trinkfreudig, hilfsbereit, gemütlich und humorvoll über die Dänen vermehrt angeführt. Insgesamt zeigt sich, dass die häufigsten Nennungen ein positives Gesamtbild von den Menschen in Dänemark zeichnen. Die deutschen Daten zeigen demnach ein sehr einheitliches Bild von typischen dänischen Frauen als blond, freundlich, hübsch, selbstbewusst, offen, modisch/modebewusst, entspannt und sportlich.

Der typische Däne wird ebenfalls als blond, freundlich, selbstbewusst, gelassen und sportlich wahrgenommen. Außerdem wird er als bärtig, groß, ruhig, hilfsbereit und gerne Bier bzw. Alkohol trinkend beschrieben. 
In den dänischen Daten ist das Bild der Deutschen unübersichtlicher. Die deutschen Frauen werden als höflich, zurückhaltend, freundlich und fleißig, aber auch als langweilig charakterisiert. Sie sind den Daten nach blonde, eher dicke, nicht besonders hübsche Hausfrauen mit großen Brüsten und ggf. einem Schnurrbart, die einen einerseits als eher hässlich und altmodisch und andererseits als bunt eingestuften Kleidungs- und Haarstyle pflegen. Das Bild vom typischen deutschen Mann ist ähnlich: höflich, arbeitsam, korrekt und zurückhaltend. Er hat einen Schnurrbart, ist groß und dick ggf. mit Bierbauch und hat ebenfalls einen eher hässlichen Kleidungsstil (Lederhosen) und eine langweilige Frisur (Bundesligahaare). Die einzelnen Nennungen weisen aber eine zu geringe Häufigkeit auf, um ein eindeutiges Bild zeichnen zu können. Tendenziell überwiegen jedoch die eher negativ bewerteten Eigenschaften.

\subsubsection{Typisch dänisch - typisch deutsch}

Die dritte Frage bezog sich auf die Angabe des für die Teilnehmenden selbst als typisch dänisch bzw. deutsch Empfundenen. Die Frage wurde ebenfalls zweigeteilt in (a) das (eher) positive und (b) das (eher) negative Typische. Die Unterfragen wurden randomisiert.

Die deutschen Antworten zum typisch negativen Dänischen zeigen ein Bild von Dänemark als einem teuren Land mit hohen Steuern, Fremden-/Ausländerfeindlichkeit und Nationalstolz, das von verschlossenen, unfreundlichen, egozentrierten, nationalistischen Menschen bevölkert wird, die außerdem schlechte Autofahrer sind, schlechtes Essen haben und (zu) viel Alkohol trinken. Bei den deutschen Nennungen zu typisch dänisch, eher positiv, überwiegen Begriffe wie Freundlichkeit, Gelassenheit, Gemütlichkeit/hygge, Entspanntheit, Offenheit, Hilfsbereitschaft, Design, Hotdog, Ruhe, Landschaft und Natur. Aber auch bei dem typisch Positiven werden die Wörter Nationalstolz, Essen und Flagge genannt.

Als typisch deutsch, eher negativ, überwiegen eindeutig Bezeichnungen der deutschen Sprache als eine unschöne, grammatisch komplizierte und schwere/schwer zu lernende Sprache. Auch die fehlenden Fremdsprachenkenntnisse der Deutschen (insbesondere für Englisch) werden hier häufig genannt. Die anderen Nennungen sind eher heterogen, beziehen sich jedoch meist auf das Aussehen (langweiliger/hässlicher/altmodischer Kleidungsstil), das Benehmen (arrogant, aggressiv, autoritätsgläubig, formell, fehlender Humor, können nicht Auto fahren), gesellschaftliche Phänomene (Bürokratie, Regeln, Ungleichheit, Hierarchie, Autobahn) und Lebensmittel (schlechtes Essen, Würstchen in vielen Varianten, Sauerkraut).

Die Antworten zu typisch deutsch, (eher) positiv, sind ebenso relativ heterogen. Folgende Nennungen kommen häufig vor: billige Waren/Produkte und niedrige Preise, Qualität, Freundlichkeit, Ordnung, Gründlichkeit, Höflichkeit, Offenheit, Pünktlichkeit, Hilfsbereitschaft, Autos, Essen, Würstchen und Bier (beides in mehreren Varianten), Kultur, Oktoberfest und Verkehr. 


\subsubsection{Stereotype Eigenschaften für die Werbung}

Die vierte und letzte Frage ${ }^{9}$ erfasste die typischen deutschen bzw. dänischen Eigenschaften, die die Teilnehmenden für geeignet halten, um ein dänisches Produkt in Deutschland bzw. ein deutsches Produkt in Dänemark zu vermarkten:

- Deutsche Teilnehmende: Eine große dänische Firma bittet Sie, eine Werbekampagne in Deutschland zu machen. Welche typischen dänischen Eigenschaften würden Sie in einer solchen Kampagne einsetzen, um ein dänisches Produkt in Deutschland zu verkaufen? Sie können die Frage gerne mit der Angabe eines konkreten Produkts beantworten.

- Dänische Teilnehmende: Eine große deutsche Firma bittet Sie, eine Werbekampagne in Dänemark zu machen. Welche typischen deutschen Eigenschaften würden Sie in einer solchen Kampagne einsetzen, um ein deutsches Produkt in Dänemark zu verkaufen? Sie können die Frage gerne mit der Angabe eines konkreten Produkts beantworten.

Mit der Frage sollen positive Eigenschaften erfasst werden, von denen angenommen wird, dass sie verkaufsfördernd für die Produkte des jeweils anderen Landes eingesetzt werden können.

Bei den typischen dänischen Eigenschaften für die Vermarktung von dänischen Produkten in Deutschland überwiegen Nennungen wie Design, Qualität, Landschaft, Natur, Strand, Naturverbundenheit, nordisch, gemütlich/Gemütlichkeit, Gelassenheit und Flagge. Die dänischen Teilnehmenden sind sich einig, dass Qualität als die typische Eigenschaft für deutsche Produkte schlechthin gilt. Weitere Nennungen, die jedoch eine viel geringere Häufigkeit aufweisen, sind Gründlichkeit, Autos, Effektivität und Haltbarkeit.

\subsection{Positive und negative Wertung von Stereotypen}

Ein bedeutsames Ergebnis der Datenauswertung ist, dass Stereotype von den Teilnehmenden im Hinblick auf ihre Einordnung als negativ oder positiv unterschiedlich bewertet werden. Stereotype sind daher im Hinblick auf ihre Wertung differenziert zu betrachten: Dieselben Stereotype können gleichzeitig positiv und negativ wahrgenommen werden. Sowohl bei der Frage nach positiven Eigenschaften (4) als auch bei der Frage nach negativen Eigenschaften (5) werden Begriffe wie Gelassenheit, zurückhaltend und Dannebrog genannt. Diese Konzepte werden von den Befragten also anscheinend positiv wie negativ bewertet. Auch in den dänischen Daten wurden einige Nennungen (z. B. Essen, Würstchen, Ordnung, Autobahn) sowohl negativ als auch positiv eingestuft. Darin spiegelt sich die subjektive Beurteilung der Teilnehmenden wider, die auf ihrer individuellen Erfahrung mit den jeweiligen Phänomenen basiert.

Diese Tendenz zeigen die Daten vor allem im Hinblick auf eine fließende Einordnung von Patriotismus bzw. Liebe zum Heimatland als positives Merkmal bis hin zum Nationalstolz als negatives typisch dänisches Merkmal. Auch bei der Nennung Dannebrog wird diese Uneinigkeit deutlich. Eine ähnliche Streuung ist in der Beurteilung der dänischen Gelassenheit zu beobachten, die von den deutschen Befragten überwiegend als positiv angesehen wird, bei

\footnotetext{
${ }^{9}$ Diese Frage ist eine freie Variation einer Frage zu Stereotypen in der Fernsehwerbung, die auf die Arbeit von Sonja Vandermeeren für das SMiK-Projekt zurückgeht.
} 
einigen aber als zu locker bis hin zu unzuverlässig als eine Art komm 'ich heut 'nicht, komm ' ich morgen-Einstellung wahrgenommen wird. Stereotype entfalten sich also sehr unterschiedlich und können daher nicht immer strikt an einem Pol verortet werden.

\section{Textanalysen des SMiK-Projekts}

Um das Vorkommen von Stereotypen anhand einer anderen Methode im Sinne des Methodenpluralismus zu überprüfen, wurden die Korpora des Projekts Deutscher Wortschatz (Universität Leipzig) und die für das Projekt erstellten Presse- und Forenkorpora des Dresden Center for Digital Linguistics (Universität Dresden) hinzugezogen. Das Ziel der Einbindung der Methode der Textanalyse ist die Ermittlung und Belegung der sprachlichen Manifestation nationaler Stereotype in Texten der deutschen und dänischen Gegenwartssprache. Die Erfassung und Beschreibung der deutsch-dänischen Heterostereotype in der Sprache erfolgt auf der Text-, Satz-, Wortgruppen- und Wortebene. Die Ausgangsfragen bei den Textanalysen sind, welche Stereotype auf welche Art und Weise versprachlicht werden sowie ob und wie sich die kulturellen Stereotype in den Köpfen der Menschen in sprachlicher Form in geschriebenen Texten manifestieren.

\subsection{Stereotype in Foren}

An dieser Stelle soll die Analyse in ausgewählten Foren ${ }^{10}$ näher betrachtet werden. In den Forenanalysen werden die geschriebene, überregionale Standardsprache und die geschriebene regionale Umgangssprache untersucht, das heißt, dass auch nicht-standardsprachliche Realisierungen von Stereotypen berücksichtigt werden. Bei den Forenanalysen wird untersucht, welche Stereotype sich in welcher sprachlichen Form in geschriebenen Texten manifestieren. Die Quellen der Forenanalysen waren Das Dänemark Forum, das DK-Forum, Handelsblatt online, Spiegel online, TAZ, WAZ, Die Welt sowie Zeit online.

Die Texte in den Foren wurden auf zweierlei Art analysiert. Im Rahmen der systematisierten Recherche wurde zum einen nach den Morphemen \{deutsch\} und \{dän\} bzw. \{tysk\} und \{dan\} in Kombination mit syntaktischen Formen (sogenannte Haben-, Tun-, SeinKonstruktionen) oder Prädikationen des Typs Der Däne ist/Die Dänin ist x, (Alle) Dänen/Däninnen sind $x$ gesucht (cf. dazu auch Kilian in diesem Heft). Zum anderen konnte in den Forentexten überprüft werden, ob sich die zuvor in der bundesweiten Fragebogenaktion ermittelten Eigenschaften wie z. B. freundlich oder blond mit den oben genannten Morphemen verknüpft in der geschriebenen Sprache manifestieren. Die in den Foren ermittelten Belege wurden intersubjektiv dahingehend ausgewertet, ob es sich um ein sprachlich manifestiertes Stereotyp handelt. Im Rahmen der manuellen Recherche wurden ebenso die Morpheme \{deutsch\} und \{dän\} bzw. \{tysk\} und \{dan\} zur Operationalisierung genutzt. In dieser Analyse wurden Korrelationen mit diskursspezifischen Formen in Zusammenhang mit zeitgeschichtlichen Ereignissen (wie dem Referendum zur Euro-Einführung in Dänemark 2000 oder der Einführung von Grenzkontrollen 2011) aufgedeckt. Als Themenfelder, die aus den Ereig-

\footnotetext{
10 Die Texte aus den Foren wurden von Noah Bubenhofer am Dresden Center for Digital Linguistics (http://linguistik.zih.tu-dresden.de/ [04.10.2015]) gecrawlt und als Korpus für die qualitative Textanalyse aufbereitet.
} 
nissen zusammengestellt wurden, ergaben sich u. a. Arbeitswelt, Demokratie, Essen, Mentalität und Gesundheitssystem.

Bei der Suche nach sprachlichen Stereotypen in den Foren wurde u. a. untersucht, welche lexikalischen Einheiten mit der Prädikativstruktur Der Däne ist korrelieren (cf. dazu auch Kilian in diesem Heft). Als Ergebnis konnte unter anderem festgehalten werden, der Däne sei zufrieden, hilfsbereit, ein Milch-Trinker, lernfähig, ausländerfeindlich, ein Pølse-Esser, freundlich und flexibel beim Hauskauf. Bei der Suche nach der Prädikativstruktur Dänen sind wurden Begriffe wie freundlich, nett, hilfsbereit, glücklich, zufrieden oder locker gefunden. Die Ähnlichkeit zu den Fragebogendaten ist hier zwar sichtbar, die Häufigkeiten der Nennungen aus dem Fragebogen und aus den Foren zeigen aber keine eindeutigen Relationen. Bemerkenswert ist, dass die dänische Nationalität gleichzeitig mit drei anderen Nationalitäten in eine direkte Verbindung gebracht wird: Die Dänen werden die Italiener, die Schweizer und die Franzosen des Nordens genannt, wobei diesen Nationalitäten (positive) stereotype Eigenschaften unterstellt werden, die den Dänen durch den Vergleich ebenfalls zugeschrieben werden. Wird in die Forensuche außerdem das Wort typisch eingegeben, tauchen Begriffe wie Gemütlichkeit, Hotdogs, Gelassenheit, hygge und Strand auf. Diese Angaben decken sich zum Teil mit den Fragebogenantworten aus Frage 1 (assoziatives Faktenwissen). Im Zusammenhang mit der Forenanalyse soll auf die Relevanz der Ebene der Sprachnormen hingewiesen werden, wie diese im Modell der Existenzweisen von Sprache bei Kilian (2003: 161) dargestellt wird. Innerhalb der standardsprachlichen Normen erscheinen sprachlich gefasste Stereotype oft sublimiert bzw. überformt; in umgangssprachlichen Normen/Registern, wie man sie etwa in Foren vorfindet, gelten hingegen andere Sprachverkehrsnormen, da sprachlich gefasste Stereotype bisweilen anders sprachlich kodiert sind.

\subsection{Das Vorkommen der Stereotype aus den Fragebogen in den Korpora}

Von den 7400 Forentreffern zu freundlich wurden 205 als stereotyp eingestuft (3\%). Diese Zahl kann aufgrund der unterschiedlichen Methoden zwar nicht direkt mit den Fragebogenergebnissen in einen Vergleich gebracht werden, zeigt aber dennoch, dass nur ein vergleichsweise geringer Anteil der insgesamt großen Anzahl an Textbelegen zu freundlich in den Foren als Stereotype wahrgenommen wird. Die Eigenschaft blond tauchte in den Forentexten insgesamt 458-mal auf, ein Stereotyp konnte aber ebenso nur in vergleichsweise wenigen Belegen (15 Belege, 3 \%) festgestellt werden. Beispielhaft werden die Stereotype in Forentexten folgendermaßen geäußert:

- Ich komme gerne nach Dänemark, weil das Land so toll ist und die Dänen so freundlich und liebenswert sind.

- $\quad$ Blonde Wikinger (sehen aus wie Asterix), bärtig und mit Hornhelm auf, Pfeil und Bogen, Schwerter und lautlose Schiffe mit rot-weiss gestreiften Segeln.

Abschließend soll das Vorkommen einiger Nennungen aus der letzten Fragebogenfrage im Forenkorpus beleuchtet werden. Sehr auffällig in den Antworten ist die häufige Nennung von Design. Dänisches Design scheint ein feststehender Begriff in den Vorstellungen der deutschen Befragten zu sein. In insgesamt 558 Fragebogenantworten kommt Design 115-mal vor, davon 69-mal in den Antworten zu Frage 4. Das ist eine vergleichsweise hohe Anzahl, die 
sich auch in den Forentexten mit 193 Nennungen widerspiegelt (vor allem im Vergleich dazu, dass die in den Fragebogenantworten sehr häufig genannten Begriffe blond 15- und freundlich 205-mal in den Foren auftauchen). Die Versprachlichung dieser Stereotype erfolgt sehr differenziert in den Foren. Hier tauschen sich die Nutzerinnen und Nutzer über das Thema Design inhaltlich detailliert aus und nennen dänische Designer namentlich wie etwa Arne Jacobsen, Fritz Hansen und Jacob Jensen. In einer Fragebogenerhebung ist eine solche elaborierte inhaltliche Auseinandersetzung dagegen nicht vorgesehen.

Generell ist anzumerken, dass sich die Stereotype, die in den Foren sprachlich realisiert werden, teilweise mit den in den Fragebogenantworten erfassten Vorstellungen in den Köpfen der Menschen decken. Es ergibt sich also ein ähnliches Bild, die Relation zwischen den kulturellen Stereotypen in den Köpfen der Menschen und den Stereotypen in Texten ist jedoch nicht linear, da sich die Stereotype nicht in derselben Häufigkeit in den Texten wie in den Fragebogendaten manifestieren.

Der Vergleich der Fragebogendaten und den Daten aus den Forenkorpora zeigt uns deutlich, dass mit nur einer einzigen Methode kein komplettes Bild von Nationalstereotypen gezeichnet werden kann. Für ein vollständige(re)s Bild ist es daher notwendig, einen Methodenpluralismus einzusetzen. Im SMiK-Projekt haben wir exepmlarisch gezeigt, wie nationale Stereotype durch die Anwendung verschiedener Methoden erfasst werden können.

\section{Diskussion und Ausblick}

Im Rahmen des SMiK-Projekts wurden verschiedene Untersuchungen, darunter Fragebogenaktionen und Textanalysen, durchgeführt. Dieser Methodenmix machte es möglich, ein differenziertes Bild über deutsch-dänische nationale Stereotype zu gewinnen. Dass die assoziativen Stereotype als Weltansichten (cf. Kilian 2015) in den Köpfen der Menschen dabei nicht immer den versprachlichten Stereotypen in Texten entsprechen, ist ein wichtiges Ergebnis. Darin zeigt sich: Was Menschen tatsächlich denken, kann stark davon abweichen, was in Medien oder Foren geschrieben wird. Das SMiK-Projekt lenkt daher den Fokus auf diese verschiedenen Formen der Manifestation von Stereotypen, um ein ganzheitliches Bild über deutsch-dänische Stereotype zu erhalten. Der Vergleich der Ergebnisse aus den unterschiedlichen Untersuchungen wirft außerdem die Frage auf: Wie werden Stereotype tradiert, wenn nicht über die (geschriebene) Sprache?

Die Analyse der Antworten aus der Fragebogenuntersuchung „Typisch deutsch - typisch dänisch“ zeigt uns, dass sich viele Nennungen auf das angenommene Aussehen und die angenommenen Charaktereigenschaften sowie Alltagsphänomene wie Essen, Umgangsformen und kulturspezifische Phänomene der jeweils anderen Nationalität beziehen. Einige Eigenschaften (z. B. nicht ordentlich Auto fahren können) und Artefakte (z. B. Essen, Bier, Würstchen) werden sowohl von den Dänen als typisch Deutsch als auch von den Deutschen als typisch Dänisch angesehen. Die Analyse der Fragebogendaten ergibt ein deutlich differenzierteres und komplizierter zu erfassendes Dänemark- bzw. Deutschlandbild als die bisherige Forschungsliteratur aufzeichnet. Die durch die Fragebogenuntersuchung „Typisch deutsch - typisch dänisch“ festgestellten Stereotype sind auf beiden Seiten vielfältiger als bisher angenommen. 
Das Dänemarkbild der Deutschen ist mit wenigen Ausnahmen sehr positiv und es wird insbesondere von Vorstellungen über den erholsamen Urlaub am Meer, Gelassenheit, Gemütlichkeit (hygge; cf. aber Anm. 4) und freundliche Menschen mit blonden Haaren geprägt. Den Deutschen haftet zwar immer noch das Bild von eher langweiligen und unscheinbaren Menschen an, sie werden aber auch als freundlich, höflich und ordentlich beschrieben.

Eine absolute Dominanz des Zweiten Weltkrieges und des Nazismus im Deutschlandbild der Dänen kann in den Daten nicht mehr festgestellt werden. Nennungen aus diesem Themenbereich kommen fast ausschließlich in den Antworten zu der ersten Frage vor und sind somit eher als assoziatives bzw. historisches Faktenwissen anzusehen, als dass es sich hierbei um eigentliche Stereotype handelt. Solches Wissen bildet allerdings das Potenzial, aus dem sich wieder Stereotype entwickeln bzw. aus dem alte Stereotype wiederbelebt werden können.

Von besonderem Interesse für die fremdsprachendidaktische Perspektive im SMiK-Projekt ist die Relation zwischen der Auffassung von der deutschen Sprache als Unterrichtsfach und dem dadurch geprägten Bild von Deutschland in Dänemark (cf. Müller 2016). Die bei den dänischen Teilnehmenden häufig vorkommende Nennung der deutschen Sprache als etwas typisch Negatives stellt unserer Meinung nach einen Anlass zum Nachdenken über den Unterricht in Deutsch als Fremdsprache in Dänemark dar. Die Teilnehmenden haben alle Deutschkenntnisse - zumindest für die passiven Sprachfertigkeiten sogar teilweise recht gute - und die negative Stereotypisierung der Sprache in den Antworten deutet auf eine ernstzunehmende Problematik im Umgang mit der deutschen Sprache im dänischen Ausbildungssystem hin.

Stereotype, so wie sie im SMiK-Projekt als Denk- und Handlungsmuster definiert werden, sind ein wichtiger Bestandteil unserer Weltansichten. Das Reflektieren über Stereotype sollte daher unserer Meinung nach ein fester Bestandteil von fremdsprachendidaktischen Überlegungen sein. Die Ergebnisse aus der Fragebogenuntersuchung wurden bereits in Unterrichtsmaterialien zur Bewusstmachung von nationalen Stereotypen für Deutsch und Dänisch als Fremdsprachen (cf. Müller/Hallsteinsdóttir 2016) sowie in Ratgebermaterialien für die interkulturelle Kommunikation umgesetzt (cf. Müller/Hallsteinsdóttir 2016 in diesem Heft).

\section{Literatur}

Bolten, Jürgen (2006): „Die Entwicklung von Nationalstereotypen im Globalisierungsprozess. Hypothesen zum Auftakt einer international durchgeführten Langzeituntersuchung zu Veränderungen des Deutschlandbildes bei Studierenden“. Zeitschrift für Interkulturellen Fremdsprachenunterricht 11/3. http://tujournals.ulb.tu-darmstadt.de/index.php/zif /article/view/393/381 [04.10.2015].

Bolten, Jürgen (2007): Interkulturelle Kompetenz. Erfurt: Landeszentrale für politische Bildung Thüringen. www.db-thueringen.de/servlets/DerivateServlet/Derivate20394/interkulturellekompetenz.pdf [04.10.2015].

Bolten, Jürgen/Weißflog, Anita (2005): Weltweite Befragung zum internationalen Interesse an Themen zur deutschen Kultur 2005. Statistische Ergebnisse der Umfragebögen. www.interkulturelles-portal.de/c/document_library/get_file?p_1_id=10237\&folder $\mathrm{Id}=218351$ \&name=DLFE-14512.pdf [04.10.2015]. 
Fink, Matthias C. (2003): „Das Deutschlandbild in dänischen Lehrwerken für den Deutschunterricht in der Folkeskole“. Informationen Deutsch als Fremdsprache 30/5: 476-488.

Hallsteinsdóttir, Erla (2013): „Möglichkeiten textbezogener Sprachdidaktik“. Deutsch als Fremdsprache 2/2013: 93-101.

Hallsteinsdóttir, Erla (2015a): Ergebnisse aus der SMiK-Fragebogenuntersuchung zu „typisch deutsch - typisch dänisch" || Resultater fra SMiK-spørgeskemaundersøgelsen om "typisk dansk - typisk tysk". Odense: SMiK-Projekt.

www.stereotypenprojekt.eu/projektresultate-r-1/fragebogen-typisch-deutsch-typischdänisch-spørgeskema-typisk-dansk-typisk-tysk/ [04.10.2015].

Hallsteinsdóttir, Erla (2015b): „Kontrastive Wortbildung im Sprachsystem, Sprachgebrauch und in der Sprachkompetenz". In: Kilian, Jörg/Eckhoff, Jan (eds.): Deutscher Wortschatz beschreiben, lernen, lehren. Beiträge zur Wortschatzarbeit in Wissenschaft, Sprachunterricht, Gesellschaft. Frankfurt a. M. etc., Lang: 351-368.

Hallsteinsdóttir, Erla (2016): „Deutsch-dänische Nationalstereotype in der Sprache“. In: Hallsteinsdóttir, Erla et al. (eds.). Perspektiven der Stereotypenforschung. Frankfurt a. M. etc., Lang: $35-57$.

Hallsteinsdóttir, Erla/Farø, Ken (2010): „Interlinguale Phraseologie: Theorie, Praxis und Perspektiven / Interlingual Phraseology: Theory, Practice and Perspectives“. Yearbook of Phraseology: 125-158.

Hallsteinsdóttir, Erla/Kilian, Jörg (eds.) (2015): SMiK-Posterpräsentation von der Konferenz $\{D E U T S C H\}$ und $\{D A ̈ N I S C H\}$ im Stereotyp: Stereotypenwelten und ihre sprachlichkulturellen Konstituierungsformen, 25.-27. Februar 2015 an der Süddänischen Universität in Odense || SMiK-Plakate fra konferencen $\{D A N S K\}$ og \{TYSK\} i stereotyper: stereotypeuniverser og deres sproglig-kulturelle konstitueringsformer, 25.-27. februar 2015 ved Syddansk Universitet i Odense. Odense, SMiK-Projekt.

www.stereotypenprojekt.eu/projektresultate-r-1/smik-publikationer-n/smik-publikationen$\mathrm{r} /[04.10 .2015]$.

Heier, Anke (2016): „Dänischer Rechtspopulismus und Tyskertøser - Zu nationalen Stereotypen in ein- und zweisprachigen Wörterbüchern“. In: Hallsteinsdóttir, Erla et al. (eds.). Perspektiven der Stereotypenforschung. Frankfurt a. M. etc., Lang: 15-34.

Henningsen, Bernd (1991): „Der Deutsche wird nie ein guter Däne. Zum Bild der Deutschen in Dänemark“. In: Trautmann, Günter (ed.): Die häßlichen Deutschen: Die Deutschen im Spiegel der westlichen und östlichen Nachbarn. Darmstadt, Wissenschaftliche Buchgesellschaft: $167-180$.

Henningsen, Bernd (1996): „Das dänisch-deutsche Verhältnis in politischer Perspektive: Desinteresse, Projektionen, Ängste, Wiederholungszwänge“. In: Süssmuth, Hans (ed.):

Deutschlandbilder in Dänemark und England, in Frankreich und den Niederlanden. Baden-Baden, Nomos: 141-155.

Kilian, Jörg (2001): „Kritische Semantik. Für eine wissenschaftliche Sprachkritik im Spannungsfeld von Sprachtheorie, Sprachnorm, Sprachpraxis“. Zeitschrift für Germanistische Linguistik 29: 293-318.

Kilian, Jörg (2003): „Wörter im Zweifel. Ansätze einer linguistisch begründeten kritischen Semantik“. Linguistik online 16/4: 159-170. 
Kilian, Jörg (2015): „Von blonden Däninnen aus deutscher Sicht. Nationale Stereotype und didaktische Sprachkritik - ein Zwischenbericht aus einem laufenden Forschungsprojekt“". In: Peschel, Corinna/Runschke, Kerstin (eds.): Sprachvariation und Sprachreflexion in interkulturellen Kontexten. Frankfurt a. M. etc., Lang: 155-182.

Lammers, Karl Christian (2000): „Dänemarks Deutschlandbild und die Entwicklung seiner politischen Beziehungen zu den beiden deutschen Staaten“. In: Bohn, Robert/Elvert, Jürgen/Lammers, Karl Christian (eds.): Deutsch-skandinavische Beziehungen nach 1945. Stuttgart, Franz Steiner: 57-66.

Langer, Roy (2000): Zwischen Gefühl und Vernunft - zur Darstellung Deutschlands in dänischen Mediendiskursen. Frederiksberg: Dissertation an der Wirtschaftsuniversität Kopenhagen.

Langer, Roy (2000a): „Zum Änderungspotential nationaler Images: Dänische Medien über Deutschland und Deutsche“. In: Müssener, Helmut/Kirsch, Frank-Michael (eds.): Nachbarn im Ostseeraum unter sich. Vorurteile, Klischees und Stereotypen in Texten. Stockholm, Almquist \& Wiksell: 90-105.

Langer, Roy (2003): Die Darstellung Deutschlands in dänischen Medien. Eine Mediendiskursanalyse. Wiesbaden: Deutscher Universitäts-Verlag.

Meyer, Britta (2014): Typically Danish - National Stereotypes of the Danes and their Meaning within Marketing Communication. Odense: Unpubl. Abschlussarbeit an der Süddänischen Universität in Odense.

Ministry for Economic and Business Affairs (2007): Action Plan for the Global Marketing of Denmark. Kopenhagen.

Müller, Katarina Le (2016): „Thematisierungen von Sprache in der deutsch-dänischen interkulturellen Kommunikation“. In: Hallsteinsdóttir, Erla et al. (eds.): Perspektiven der Stereotypenforschung. Frankfurt a. M. etc., Lang: 59-76.

Müller, Katarina Le/Hallsteinsdóttir, Erla (2016): „Stereotype im Fremdsprachenunterricht“. In: Hallsteinsdóttir, Erla et al. (eds.): Perspektiven der Stereotypenforschung. Frankfurt a. M. etc., Lang: 233-256.

Müller, Kathrin (2005): Dänemarks Selbst- und Fremdbild im Kontext der europäischen Integration. Eine Medieninhaltsanalyse. Kiel: Dissertation an der Christian-AlbrechtsUniversität zu Kiel.

Øhrgaard, Per (1996): „Das Bild des Deutschen in der dänischen Literatur - Einige Beispiele“. In: Süssmuth, Hans (ed.): Deutschlandbilder in Dänemark und England, in Frankreich und den Niederlanden. Baden-Baden, Nomos: 194-210.

Østergård, Uffe (1991): „Feindbilder und Vorurteile in der dänischen Öffentlichkeit“. In: Trautmann, Günter (ed.): Die häßlichen Deutschen: Die Deutschen im Spiegel der westlichen und östlichen Nachbarn. Darmstadt, Wissenschaftliche Buchgesellschaft: 145-166.

Østergård, Uffe (1996): „Das Deutschlandbild in Dänemark“. In: Süssmuth, Hans (ed.): Deutschlandbilder in Dänemark und England, in Frankreich und den Niederlanden. Baden-Baden, Nomos: 170-193.

ReD Associates (2006): Denmark: Perceptionsanalyse og anbefalinger til en offensiv global markedsføring af Danmark. Kopenhagen.

http://pure.au.dk/portal/files/11064/Appendix_Danmark_TM_- 
_Perceptionsanalyse_og_anbefalinger_til_en_offensiv_global_markedsf_ring_af_Danmark _ReD_Associates.pdf [04.10.2015].

Rerup, Lorenz (1996): „Deutschlandbilder in Dänemark“. In: Süssmuth, Hans (ed.): Deutschlandbilder in Dänemark und England, in Frankreich und den Niederlanden. Baden-Baden, Nomos: 170-193.

Ruby, Jørn (1996): „Zum aktuellen Deutschlandbild in der veröffentlichten dänischen Meinung“. In: Süssmuth, Hans (ed.): Deutschlandbilder in Dänemark und England, in Frankreich und den Niederlanden. Baden-Baden, Nomos: 211-214.

Schramm, Moritz (2010): „Tysk som trend: forandringer i det danske tysklandsbillede“. In: Skovgaard Andersen, Mette et al. (eds.): Tysk nu. Konference om tysk sprog og kultur $i$ offentlighed, forskning og undervisning $i$ Danmark. Kopenhagen/Roskilde, Københavns Universitet, Roskilde Universitet, IISV \& CBS: 34-37.

Schulze, Sylvia (2010): „Das Lehrwerk als bildprägende Instanz? Die Landesbilder von Großbritannien und den USA bei Schülerinnen und Schülern unter dem Einfluss des Lehrwerks". ForumSprache 4: 43-58.

Skovgaard Andersen, Mette (2010): „Tysk i krise - tysk i det danske uddannelsessystem“. In: Skovgaard Andersen, Mette et al. (eds.): Tysk nu. Konference om tysk sprog og kultur $i$ offentlighed, forskning og undervisning i Danmark. Kopenhagen/Roskilde, Københavns Universitet, Roskilde Universitet, IISV \& CBS: 4-15.

Thomsen, Karina/Thomsen Kamilla (2008): Tyskfagets image blandt 7. og 9. klasses elever. Aalborg: Unpubl. Abschlussarbeit an der Aalborg Universität. http://projekter.aau.dk/projekter/files/14411565/Speciale_020608_23xx.doc [04.10.2015].

Vandermeeren, Sonja (2013): „Kulturdimensionen und Stereotype“. In: Schneider-Wiejowski, Karina/Kellermeier-Rehbein, Birte/Haselhuber, Jakob (eds.). Vielfalt, Variation und Stellung der deutschen Sprache. Berlin/Boston, de Gruyter: 563-578.

Vandermeeren, Sonja (2016): „Kulturdimensionen und Stereotype: Begriffserläuterungen und -operationalisierungen“. In: Hallsteinsdóttir, Erla et al. (eds.). Perspektiven der Stereotypenforschung. Frankfurt a. M. etc., Lang: 163-183.

Vandermeeren, Sonja/Hofmann, Annika (2015): „Wie lassen sich Stereotype mit Kulturdimensionen verknüpfen? Eine empirische Untersuchung in Deutschland und Dänemark“" In: Andrzejewska, Joanna (ed.): Das Fremde in den interkulturellen Untersuchungen. Erfurt, City Druck \& Verlag: 127-147.

Vandermeeren, Sonja/Hofmann, Annika (2016): „Deutsch-dänische Kulturdimensionen und Stereotype: Ergebnisse einer Fragebogenuntersuchung mit Danfoss-Mitarbeitern“. In: Hallsteinsdóttir, Erla et al. (ed.). Perspektiven der Stereotypenforschung. Frankfurt a. M. etc., Lang: 185-206. 